\title{
Some inequalities for interval-valued functions on time scales
}

\author{
Dafang Zhao ${ }^{1,2} \cdot$ Guoju Ye $^{1} \cdot$ Wei Liu ${ }^{1} \cdot$ Delfim F. M. Torres ${ }^{3}$
}

Submitted: 20-Dec-2017 / Revised: 31-Aug-2018 / Accepted: 11-Sept-2018

\begin{abstract}
We introduce the interval Darboux delta integral (shortly, the $I D \Delta$-integral) and the interval Riemann delta integral (shortly, the IR $\Delta$-integral) for interval-valued functions on time scales. Fundamental properties of $I D$ and $I R \Delta$-integrals and examples are given. Finally, we prove Jensen's, Hölder's and Minkowski's inequalities for the $I R \Delta$-integral. Also, some examples are given to illustrate our theorems.
\end{abstract}

Keywords interval-valued functions · time scales . Jensen's inequality · Hölder's inequality · Minkowski's inequality

\section{Introduction}

Interval analysis was initiated by Moore for providing reliable computations 27. Since then, interval analysis and interval-valued functions have been extensively studied both in mathematics and its applications: see,

Dafang Zhao

E-mail: dafangzhao@163.com

Guoju Ye

E-mail: yegj@hhu.edu.cn

Wei Liu

E-mail: liuw626@hhu.edu.cn

Delfim F. M. Torres

E-mail: delfim@ua.pt

${ }^{1}$ College of Science, Hohai University, Nanjing, Jiangsu 210098, P. R. China.

${ }^{2}$ School of Mathematics and Statistics, Hubei Normal University, Huangshi, Hubei 435002, P. R. China.

${ }^{3}$ Center for Research and Development in Mathematics and Applications (CIDMA),

Department of Mathematics, University of Aveiro, 3810-193 Aveiro, Portugal. e.g., 2, 11, 12, 14, 15, 21, 23, 25, 28, 29, 30, 34, 38, 42, Recently, several classical integral inequalities have been extended to the context of interval-valued functions by Chalco-Cano et al. 9, 10, Costa [13], Costa and RománFlores [16], Flores-Franulič et al. [20, Román-Flores et s al. 32,33 .

Motivated by [8,13,32, we introduce the $I D$ and IR $\Delta$-integrals, and present some integral inequalities on time scales. A time scale $\mathbb{T}$ is an arbitrary nonempty closed subset of the real numbers $\mathbb{R}$ with the subspace topology inherited from the standard topology of $\mathbb{R}$. The theory of time scales was born in 1988 with the $\mathrm{Ph} . \mathrm{D}$. thesis of Hilger 22. The aim is to unify various definitions and results from the theories of discrete and continuous dynamical systems, and to extend them to more general classes of dynamical systems. It has undergone tremendous expansion and development on various aspects by several authors over the past three decades: see, e.g., 3, 4, 5, 6, 18, 19, 35, 37, 39, 40, 41].

In 2013, Lupulescu introduced the Riemann $\Delta$-integral for interval-valued functions on time scales and presented some of its basic properties 24. Nonetheless, to our best knowledge, there is no systematic theory of integration for interval-valued functions on time scales. In this work, in order to complete the theory of $I R$ $\Delta$-integration and improve recent results given in 8 , 13, 32, we introduce the $I D \Delta$-integral and the $I R \Delta$ integral on time scales. We show that the $I D \Delta$-integral ia a generalization of the $I R \Delta$-integral. Also, some basic properties for the $I D$ and $I R \Delta$-integrals, and some examples, are given. Finally, we present Jensen's inequality, Hölder's inequality and Minkowski's inequality for the $I R \Delta$-integral. Some celebrated inequalities are derived as consequences of our results.

The paper is organized as follows. After a Section 2 of preliminaries, in Section 3 the $I D$ and $I R \Delta$-integrals 
for interval-valued functions are introduced. Moreover, some basic properties and examples are given. In Section 4, we prove Jensen's, Hölder's and Minkowski's inequalities for the general $I R \Delta$-integral. We end with Section 5 of conclusions.

\section{Preliminaries}

In this section, we recall some basic definitions, notations, properties and results on interval analysis and the time scale calculus, which are used throughout the paper. A real interval $[u]$ is the bounded, closed subset of $\mathbb{R}$ defined by

$$
[u]=[\underline{u}, \bar{u}]=\{x \in \mathbb{R} \mid \underline{u} \leq x \leq \bar{u}\},
$$

where $\underline{u}, \bar{u} \in \mathbb{R}$ and $\underline{u} \leq \bar{u}$. The numbers $\underline{u}$ and $\bar{u}$ are called the left and the right endpoints of $[\underline{u}, \bar{u}]$, respectively. When $\underline{u}$ and $\bar{u}$ are equal, the interval $[u]$ is said to be degenerate. In this paper, the term interval will mean a nonempty interval. We call $[u]$ positive if $\underline{u}>0$ or negative if $\bar{u}<0$. The partial order " $\leq$ " is defined by

$$
[\underline{u}, \bar{u}] \leq[\underline{v}, \bar{v}] \Longleftrightarrow \underline{u} \leq \underline{v}, \bar{u} \leq \bar{v} .
$$

The inclusion " $\subseteq$ " is defined by

$$
[\underline{u}, \bar{u}] \subseteq[\underline{v}, \bar{v}] \Longleftrightarrow \underline{v} \leq \underline{u}, \bar{u} \leq \bar{v} .
$$

For an arbitrary real number $\lambda$ and $[u]$, the interval $\lambda[u]$ is given by

$\lambda[\underline{u}, \bar{u}]= \begin{cases}{[\lambda \underline{u}, \lambda \bar{u}]} & \text { if } \lambda>0, \\ \{0\} & \text { if } \lambda=0, \\ {[\lambda \bar{u}, \lambda \underline{u}]} & \text { if } \lambda<0 .\end{cases}$

For $[u]=[\underline{u}, \bar{u}]$ and $[v]=[\underline{v}, \bar{v}]$, the four arithmetic operators $(+,-, \cdot, /)$ are defined by

$$
\begin{aligned}
& {[u]+[v]=[\underline{u}+\underline{v}, \bar{u}+\bar{v}],} \\
& {[u]-[v]=[\underline{u}-\bar{v}, \bar{u}-\underline{v}],}
\end{aligned}
$$

$[u] \cdot[v]=[\min \{\underline{u v}, \underline{u} \bar{v}, \bar{u} \underline{v}, \overline{u v}\}, \max \{\underline{u v}, \underline{u} \bar{v}, \bar{u} \underline{v}, \overline{u v}\}]$, $[u] /[v]=[\min \{\underline{u} / \underline{v}, \underline{u} / \bar{v}, \bar{u} / \underline{v}, \bar{u} / \bar{v}\}$, $\max \{\underline{u} / \underline{v}, \underline{u} / \bar{v}, \bar{u} / \underline{v}, \bar{u} / \bar{v}\}]$, where $0 \notin[\underline{v}, \bar{v}]$.

We denote by $\mathbb{R}_{\mathcal{I}}$ the set of all intervals of $\mathbb{R}$, and by $\mathbb{R}_{\mathcal{I}}^{+}$ and $\mathbb{R}_{\mathcal{I}}^{-}$the set of all positive intervals and negative intervals of $\mathbb{R}$, respectively. The Hausdorff-Pompeiu distance between intervals $[\underline{u}, \bar{u}]$ and $[\underline{v}, \bar{v}]$ is defined by

$$
d([\underline{u}, \bar{u}],[\underline{v}, \bar{v}])=\max \{|\underline{u}-\underline{v}|,|\bar{u}-\bar{v}|\} .
$$

It is well known that $\left(\mathbb{R}_{\mathcal{I}}, d\right)$ is a complete metric space.
Let $\mathbb{T}$ be a time scale. We define the half-open interval $[a, b)_{\mathbb{T}}$ by

$$
[a, b)_{\mathbb{T}}=\{t \in \mathbb{T}: a \leq t<b\} .
$$

The open and closed intervals are defined similarly. For $t \in \mathbb{T}$, we denote by $\sigma$ the forward jump operator, i.e., $\sigma(t):=\inf \{s>t: s \in \mathbb{T}\}$, and by $\rho$ the backward jump operator, i.e., $\rho(t):=\sup \{s<t: s \in \mathbb{T}\}$. Here, we put $\sigma(\sup \mathbb{T})=\sup \mathbb{T}$ and $\rho(\inf \mathbb{T})=\inf \mathbb{T}$, where $\sup \mathbb{T}$ and $\inf \mathbb{T}$ are finite. In this situation, $\mathbb{T}^{\kappa}:=\mathbb{T} \backslash\{\sup \mathbb{T}\}$ and $\mathbb{T}_{\kappa}:=\mathbb{T} \backslash\{\inf \mathbb{T}\}$, otherwise, $\mathbb{T}^{\kappa}:=\mathbb{T}$ and $\mathbb{T}_{\kappa}:=\mathbb{T}$. If $\sigma(t)>t$, then we say that $t$ is right-scattered, while if $\rho(t)<t$, then we say that $t$ is left-scattered. If $\sigma(t)=t$ and $t<\sup \mathbb{T}$, then $t$ is called right-dense, and if $\rho(t)=$ $t$ and $t>\inf \mathbb{T}$, then $t$ is left-dense. The graininess functions $\mu$ and $\eta$ are defined by $\mu(t):=\sigma(t)-t$ and $\eta(t):=t-\rho(t)$, respectively.

A function $f:[a, b]_{\mathbb{T}} \rightarrow \mathbb{R}$ is called right-dense continuous ( $r d$-continuous) if it is right continuous at each right-dense point and there exists a finite left limit at all left-dense points. The set of $r d$-continuous function $f:[a, b]_{\mathbb{T}} \rightarrow \mathbb{R}$ is denoted by $C_{r d}\left([a, b]_{\mathbb{T}}, \mathbb{R}\right)$.

A function $f$ is said to be an interval function of $t$ on $[a, b]_{\mathbb{T}}$ if it assigns a nonempty interval

$$
f(t)=[\underline{f}(t), \bar{f}(t)]
$$

to each $t \in[a, b]_{\mathbb{T}}$. We say that $f:[a, b]_{\mathbb{T}} \rightarrow \mathbb{R}_{\mathcal{I}}$ is continuous at $t_{0} \in[a, b]_{\mathbb{T}}$ if for each $\epsilon>0$ there exists a $\delta>0$ such that

$$
d\left(f(t), f\left(t_{0}\right)\right)<\epsilon
$$

whenever $\left|t-t_{0}\right|<\delta$. The set of continuous function $f:[a, b]_{\mathbb{T}} \rightarrow \mathbb{R}_{\mathcal{I}}$ is denoted by $C\left([a, b]_{\mathbb{T}}, \mathbb{R}_{\mathcal{I}}\right)$. It is clear that $f$ is continuous at $t_{0}$ if and only if $f$ and $\bar{f}$ are continuous at $t_{0}$.

A division of $[a, b]_{\mathbb{T}}$ is any finite ordered subset $D$ having the form

$$
\mathcal{D}=\left\{a=t_{0}<t_{1}<\cdots<t_{n}=b\right\}
$$

We denote the set of all divisions of $[a, b]_{\mathbb{T}}$ by $\mathcal{D}=$ $\mathcal{D}\left([a, b]_{\mathbb{T}}\right)$.

Lemma 1 (Bohner and Peterson [6]) For every $\delta>0$ there exists some division $D \in \mathcal{D}\left([a, b]_{\mathbb{T}}\right)$ given by

$$
a=t_{0}<t_{1}<\cdots<t_{n-1}<t_{n}=b
$$

such that for each $i \in\{1,2, \ldots, n\}$ either $t_{i}-t_{i-1} \leq \delta$ or

$$
t_{i}-t_{i-1}>\delta \text { and } \rho\left(t_{i}\right)=t_{i-1} .
$$


Let $\mathcal{D}\left(\delta,[a, b]_{\mathbb{T}}\right)$ be the set of all $D \in \mathcal{D}\left([a, b]_{\mathbb{T}}\right)$ that possess the property indicated in Lemma [1. In each interval $\left[t_{i-1}, t_{i}\right)_{\mathbb{T}}$, where $1 \leq i \leq n$, choose an arbitrary point $\xi_{i}$ and form the sum

$$
S(f, D, \delta)=\sum_{i=1}^{n} f\left(\xi_{i}\right)\left(t_{i}-t_{i-1}\right)
$$

where $f:[a, b]_{\mathbb{T}} \rightarrow \mathbb{R}\left(\right.$ or $\left.\mathbb{R}_{\mathcal{I}}\right)$. We call $S(f, D, \delta)$ a Riemann $\Delta$-sum of $f$ corresponding to $D \in \mathcal{D}\left(\delta,[a, b]_{\mathbb{T}}\right)$.

Definition 1 (Bohner and Peterson [6]) A function $f:[a, b]_{\mathbb{T}} \rightarrow \mathbb{R}$ is called Riemann $\Delta$-integrable on $[a, b]_{\mathbb{T}}$ if there exists an $A \in \mathbb{R}$ such that for each $\epsilon>0$ there exists a $\delta>0$ for which

$$
|S(f, \mathcal{D}, \delta), A|<\epsilon
$$

for all $D \in \mathcal{D}\left(\delta,[a, b]_{\mathbb{T}}\right)$. In this case, $A$ is called the Riemann $\Delta$-integral of $f$ on $[a, b]_{\mathbb{T}}$ and is denoted by $A=(R) \int_{a}^{b} f(t) \Delta t$ or $A=\int_{a}^{b} f(t) \Delta t$. The family of all Riemann $\Delta$-integrable functions on $[a, b]_{\mathbb{T}}$ is denoted by $\mathcal{R}_{\left(\Delta,[a, b]_{\mathbb{T}}\right)}$.

\section{The Interval Darboux and Riemann delta integrals}

Let $f:[a, b]_{\mathbb{T}} \rightarrow \mathbb{R}_{\mathcal{I}}$ be such that $f(t)=[\underline{f}(t), \bar{f}(t)]$ for all $t \in[a, b]_{\mathbb{T}}$. We denote

$M=\sup \left\{\bar{f}(t): t \in[a, b)_{\mathbb{T}}\right\}, m=\inf \left\{\underline{f}(t): t \in[a, b)_{\mathbb{T}}\right\}$, and for $1 \leq i \leq n$,

$$
\begin{aligned}
M_{i} & =\sup \left\{\bar{f}(t): t \in\left[t_{i-1}, t_{i}\right)_{\mathbb{T}}\right\}, \\
m_{i} & =\inf \left\{\underline{f}(t): t \in\left[t_{i-1}, t_{i}\right)_{\mathbb{T}}\right\} .
\end{aligned}
$$

The lower Darboux $\Delta$-sum $L(\underline{f}, D)$ of $\underline{f}$ with respect to $D \in \mathcal{D}\left([a, b]_{\mathbb{T}}\right)$ is the sum

$$
L(\underline{f}, D)=\sum_{i=1}^{n} m_{i}\left(t_{i}-t_{i-1}\right)
$$

and the upper Darboux $\Delta$-sum $U(\bar{f}, D)$ is

$$
U(\bar{f}, D)=\sum_{i=1}^{n} M_{i}\left(t_{i}-t_{i-1}\right) .
$$

Definition 2 (The Interval Darboux delta integral) Let $I=[a, b]_{\mathbb{T}}$, where $a, b \in \mathbb{T}$. The lower Darboux $\Delta$-integral of $\underline{f}$ on $[a, b]_{\mathbb{T}}$ is defined by

$$
(D) \underline{\int}_{a}^{b} \underline{f}(t) \Delta t=\sup _{D \in \mathcal{D}\left([a, b]_{\mathbb{T}}\right)}\{L(\underline{f}, D)\}
$$

and the upper Darboux $\Delta$-integral of $\bar{f}$ on $[a, b]_{\mathbb{T}}$ is defined by

$$
\text { (D) } \bar{\int}_{a}^{b} \bar{f}(t) \Delta t=\inf _{D \in \mathcal{D}\left([a, b]_{\mathbb{T}}\right)}\{U(\bar{f}, D)\} .
$$

Then, we define the $I D \Delta$-integral of $f:[a, b]_{\mathbb{T}} \rightarrow \mathbb{R}_{\mathcal{I}}$ on $[a, b]_{\mathbb{T}}$ as the interval

$$
(I D) \int_{a}^{b} f(t) \Delta t=\left[(D) \int_{a}^{b} \underline{f}(t) \Delta t,(D) \bar{\int}_{a}^{b} \bar{f}(t) \Delta t\right] .
$$

The family of all $I D \Delta$-integrable functions on $[a, b]_{\mathbb{T}}$ is denoted by $\mathcal{I D}_{\left(\Delta,[a, b]_{\mathbb{T}}\right)}$.

Theorem 1 Let $f:[a, b]_{\mathbb{T}} \rightarrow \mathbb{R}_{\mathcal{I}}$ be such that

$$
f(t)=[\underline{f}(t), \bar{f}(t)]
$$

for all $t \in[a, b]_{\mathbb{T}}$. Then $f \in \mathcal{I D}_{\left(\Delta,[t, \sigma(t)]_{\mathbb{T}}\right)}$ and

$$
(I D) \int_{t}^{\sigma(t)} f(s) \Delta s=[\mu(t) \underline{f}(t), \mu(t) \bar{f}(t)] .
$$

Proof If $\sigma(t)=t$, then the result is obvious. If $\sigma(t)>t$, then $\mathcal{D}\left([a, b]_{\mathbb{T}}\right)$ only contains one single element given by

$$
t=s_{0}<s_{1}=\sigma(t) .
$$

Since $\left[s_{0}, s_{1}\right)=[t, \sigma(t))=\{t\}$, we have

$$
\begin{aligned}
& L(f, D)=\underline{f}(t)(\sigma(t)-t)=\mu(t) \underline{f}(t), \\
& U(f, D)=\bar{f}(t)(\sigma(t)-t)=\mu(t) \bar{f}(t) .
\end{aligned}
$$

Consequently, we obtain

$$
(I D) \int_{t}^{\sigma(t)} f(s) \Delta s=[\mu(t) \underline{f}(t), \mu(t) \bar{f}(t)] .
$$

The result is proved.

Remark 1 It is clear that if $f$ is a real-valued function, then our Definition 2 implies the definition of Darboux $\Delta$-integral introduced by [6]. We also have the following:

(1) If $\mathbb{T}=\mathbb{R}$, then Definition 2 implies the definition of Darboux interval integral introduced by Caprani et al. [8, 31.

(2) If $\mathbb{T}=\mathbb{Z}$, then each function $f: \mathbb{Z} \rightarrow \mathbb{R}_{\mathcal{I}}$ is $I D$ $\Delta$-integrable on $[a, b]_{\mathbb{T}}$. Moreover,

$$
(I D) \int_{a}^{b} f(t) \Delta t=\left[\sum_{t=a}^{b-1} \underline{f}(t), \sum_{t=a}^{b-1} \bar{f}(t)\right] .
$$

(3) If $\mathbb{T}=h \mathbb{Z}$, then each function $f: h \mathbb{Z} \rightarrow \mathbb{R}_{\mathcal{I}}$ is $I D$ $\Delta$-integrable on $[a, b]_{\mathbb{T}}$. Moreover,

$$
(I D) \int_{a}^{b} f(t) \Delta t=\left[\sum_{k=\frac{a}{h}}^{\frac{b}{h}-1} f(k h) h, \sum_{k=\frac{a}{h}}^{\frac{b}{h}-1} \bar{f}(k h) h\right] .
$$


Example 1 Suppose that $[a, b]_{\mathbb{T}}=[0,1], \mathbb{Q}$ is the set of rational numbers in $[0,1]$, and $f:[a, b]_{\mathbb{T}} \rightarrow \mathbb{R}_{\mathcal{I}}$ is defined by

$f(t)= \begin{cases}{[-1,0],} & \text { if } t \in \mathbb{Q}, \\ {[1,2],} & \text { if } t \in[0,1] \backslash \mathbb{Q} .\end{cases}$

Then,

$$
\begin{aligned}
(I D) \int_{0}^{1} f(t) \Delta t & =\left[(D) \int_{0}^{1} \underline{f}(t) d t,(D) \bar{\int}_{0}^{1} \bar{f}(t) d t\right] \\
& =[-1,2] .
\end{aligned}
$$

Example 2 Suppose that $[a, b]_{\mathbb{T}}=\left\{0, \frac{1}{3}, \frac{1}{2}, 1\right\}$ and $f:[a, b]_{\mathbb{T}} \rightarrow \mathbb{R}_{\mathcal{I}}$ is defined by

$f(t)= \begin{cases}{[-1,0],} & \text { if } t=0 \\ {\left[-\frac{1}{3}, \frac{1}{3}\right],} & \text { if } t=\frac{1}{3} \\ {\left[-\frac{1}{2}, \frac{1}{2}\right],} & \text { if } t=\frac{1}{2} \\ {[1,2],} & \text { if } t=1\end{cases}$

Then,

(D) $\int_{0}^{1} \underline{f}(t) \Delta t=(-1) \cdot \frac{1}{3}+\left(-\frac{1}{3}\right) \cdot \frac{1}{6}+\left(-\frac{1}{2}\right) \cdot \frac{1}{2}$

$$
=-\frac{23}{36}
$$

(D) $\bar{\int}_{0}^{1} \bar{f}(t) \Delta t=0 \cdot \frac{1}{3}+\frac{1}{3} \cdot \frac{1}{6}+\frac{1}{2} \cdot \frac{1}{2}=\frac{11}{36}$,

and therefore

$$
(I D) \int_{0}^{1} f(t) \Delta t=\left[-\frac{23}{36}, \frac{11}{36}\right] .
$$

Theorem 2 Let $f, g \in \mathcal{I D}_{\left(\Delta,[a, b]_{\mathbb{T}}\right)}$, and $\lambda$ be an arbitrary real number. Then,

(1) $\lambda f \in \mathcal{I D}_{\left(\Delta,[a, b]_{\mathbb{T}}\right)}$ and

$$
(I D) \int_{a}^{b} \lambda f(t) \Delta t=\lambda(I D) \int_{a}^{b} f(t) \Delta t
$$

(2) $f+g \in \mathcal{I D}_{\left(\Delta,[a, b]_{\mathbb{T}}\right)}$ and

$(I D) \int_{a}^{b}(f(t)+g(t)) \Delta t$

$\subseteq(I D) \int_{a}^{b} f(t) \Delta t+(I D) \int_{a}^{b} g(t) \Delta t$

(3) for $c \in[a, b]_{\mathbb{T}}$ and $a<c<b$,

$(I D) \int_{a}^{c} f(t) \Delta t+(I D) \int_{c}^{b} f(t) \Delta t=(I D) \int_{a}^{b} f(t) \Delta t$;

(4) if $f \subseteq g$ on $[a, b]_{\mathbb{T}}$, then

$$
(I D) \int_{a}^{b} f(t) \Delta t \subseteq(I D) \int_{a}^{b} g(t) \Delta t .
$$

Proof We only prove that part (2) of Theorem 2 holds. The other relations are obvious. Suppose that

$$
f(t)=[\underline{f}(t), \bar{f}(t)], g(t)=[\underline{g}(t), \bar{g}(t)] .
$$

Select any division $D \in \mathcal{D}\left([a, b]_{\mathbb{T}}\right)$ having the form

$$
D=\left\{a=t_{0}<t_{1}<\cdots<t_{n}=b\right\} .
$$

Then,

$$
\begin{aligned}
& \inf _{t \in\left[t_{i-1}, t_{i}\right)_{\mathbb{T}}}\{\underline{f}(t)\}+\inf _{t \in\left[t_{i-1}, t_{i}\right)_{\mathbb{T}}}\{\underline{g}(t)\} \\
& \leq \inf _{t \in\left[t_{i-1}, t_{i}\right)_{\mathbb{T}}}\{\underline{f}(t)+\underline{g}(t)\}, \\
& \sup _{t \in\left[t_{i-1}, t_{i}\right)_{\mathbb{T}}}\{\bar{f}(t)+\bar{g}(t)\} \\
& \leq \sup _{t \in\left[t_{i-1}, t_{i}\right)_{\mathbb{T}}}\{\bar{f}(t)\}+\sup _{t \in\left[t_{i-1}, t_{i}\right)_{\mathbb{T}}}\{\bar{g}(t)\},
\end{aligned}
$$

and it follows that

$$
\begin{gathered}
L(\underline{f}, D)+L(\underline{g}, D) \leq L(\underline{f}+\underline{g}, D), \\
U(\underline{f}, D)+U(\underline{g}, D) \geq U(\underline{f}+\underline{g}, D) .
\end{gathered}
$$

The intended result follows.

Example 3 Suppose that $[a, b]_{\mathbb{T}}=[0,1], \mathbb{Q}$ is the set of rational numbers in $[0,1]$, and $f, g:[a, b]_{\mathbb{T}} \rightarrow \mathbb{R}_{\mathcal{I}}$ are defined by

$$
\begin{aligned}
& f(t)= \begin{cases}{[-1,0],} & \text { if } t \in \mathbb{Q}, \\
{[1,2],} & \text { if } t \in[0,1] \backslash \mathbb{Q},\end{cases} \\
& g(t)= \begin{cases}{[0,1],} & \text { if } t \in \mathbb{Q}, \\
{[-2,-1],} & \text { if } t \in[0,1] \backslash \mathbb{Q} .\end{cases}
\end{aligned}
$$

Then

$$
f(t)+g(t)=[-1,1]
$$

for all $t \in[0,1]$. It follows that

$$
\begin{aligned}
& (I D) \int_{0}^{1} f(t) \Delta t+(I D) \int_{0}^{1} g(t) \Delta t \\
& =[-1,2]+[-2,1] \\
& =[-3,3], \\
& (I D) \int_{0}^{1}(f(t)+g(t)) \Delta t=[-1,1] .
\end{aligned}
$$

Therefore, we have

$$
\begin{aligned}
& (I D) \int_{a}^{b}(f(t)+g(t)) \Delta t \\
& \subseteq(I D) \int_{a}^{b} f(t) \Delta t+(I D) \int_{a}^{b} g(t) \Delta t .
\end{aligned}
$$

We now give Riemann's definition of integrability, which is equivalent to the Riemann $\Delta$-integral given in [24, Definition 13]. 
Definition 3 (The Interval Riemann delta integral) A function $f:[a, b]_{\mathbb{T}} \rightarrow \mathbb{R}_{\mathcal{I}}$ is called $I R \Delta$ integrable on $[a, b]_{\mathbb{T}}$ if there exists an $A \in \mathbb{R}_{\mathcal{I}}$ such that for each $\epsilon>0$ there exists a $\delta>0$ for which

$$
d(S(f, \mathcal{D}, \delta), A)<\epsilon
$$

for all $D \in \mathcal{D}\left(\delta,[a, b]_{\mathbb{T}}\right)$. In this case, $A$ is called the IR $\Delta$-integral of $f$ on $[a, b]_{\mathbb{T}}$ and is denoted by $A=$ $(I R) \int_{a}^{b} f(t) \Delta t$. The family of all $I R \Delta$-integrable functions on $[a, b]_{\mathbb{T}}$ is denoted by $\mathcal{I R}_{\left(\Delta,[a, b]_{\mathbb{T}}\right)}$.

Remark 2 Definitions 2 and 3 are not equivalent. If $f \in \mathcal{I R}_{\left(\Delta,[a, b]_{\mathbb{T}}\right)}$, then $f \in \mathcal{I D}_{\left(\Delta,[a, b]_{\mathbb{T}}\right)}$. However, the converse is not always true (see Example 1). It is clear that $f \in \mathcal{I D}_{\left(\Delta,[a, b]_{\mathbb{T}}\right)}$, but $f \notin \mathcal{I R}_{\left(\Delta,[a, b]_{\mathbb{T}}\right)}$. In fact, all bounded interval functions are $I D \Delta$-integrable, but boundedness of $f$ is not a sufficient condition for $I R$ $\Delta$-integrability. If $f$ is a continuous function, then $f \in$ $\mathcal{I R}_{\left(\Delta,[a, b]_{\mathbb{T}}\right)}$ if and only if $f \in \mathcal{I D}_{\left(\Delta,[a, b]_{\mathbb{T}}\right)}$, in which case the value of the integrals agree.

The following two theorems can be easily verified and so the proofs are omitted.

Theorem 3 If $f \in C\left([a, b]_{\mathbb{T}}, \mathbb{R}_{\mathcal{I}}\right)$, then $f \in \mathcal{I R}_{\left(\Delta,[a, b]_{\mathbb{T}}\right)}$ and

$$
(I R) \int_{a}^{b} f(t) \Delta t=\left[\int_{a}^{b} \underline{f}(t) \Delta t, \int_{a}^{b} \bar{f}(t) \Delta t\right] .
$$

Theorem 4 Let $f, g \in \mathcal{I R}_{\left(\Delta,[a, b]_{\mathbb{T}}\right)}$, and $\lambda$ be an arbitrary real number. Then,

(1) $\lambda f \in \mathcal{I R}_{\left(\Delta,[a, b]_{\mathbb{T}}\right)}$ and

$$
(I R) \int_{a}^{b} \lambda f(t) \Delta t=\lambda(I R) \int_{a}^{b} f(t) \Delta t
$$

(2) $f+g \in \mathcal{I R}_{\left(\Delta,[a, b]_{\mathbb{T}}\right)}$ and

$$
\begin{aligned}
& (I R) \int_{a}^{b}(f(t)+g(t)) \Delta t \\
& =(I R) \int_{a}^{b} f(t) \Delta t+(I R) \int_{a}^{b} g(t) \Delta t
\end{aligned}
$$

(3) for $c \in[a, b]_{\mathbb{T}}$ and $a<c<b$,

$$
(I R) \int_{a}^{c} f(t) \Delta t+(I R) \int_{c}^{b} f(t) \Delta t=(I R) \int_{a}^{b} f(t) \Delta t
$$

(4) if $f \subseteq g$ on $[a, b]_{\mathbb{T}}$, then

$$
(I R) \int_{a}^{b} f(t) \Delta t \subseteq(I R) \int_{a}^{b} g(t) \Delta t .
$$

Example 4 Suppose that $\mathbb{T}=[-1,0] \cup 3^{\mathbb{N}_{0}}$, where $[-1,0]$ is a real-valued interval and $\mathbb{N}_{0}$ is the set of nonnegative integers. Let $f:[a, b]_{\mathbb{T}} \rightarrow \mathbb{R}_{\mathcal{I}}$ be defined by

$f(t)= \begin{cases}{[t, t+1],} & \text { if } t \in[-1,0), \\ {[1,2],} & \text { if } t=0, \\ {\left[t, t^{2}+1\right],} & \text { if } t \in 3^{\mathbb{N}_{0}}\end{cases}$

If $[a, b]_{\mathbb{T}}=[-1,3]_{\mathbb{T}}$, then

$$
\begin{aligned}
& (I R) \int_{-1}^{3} f(t) \Delta t \\
& =\left[\int_{-1}^{3} \underline{f}(t) \Delta t, \int_{-1}^{3} \bar{f}(t) \Delta t\right] \\
& =\left[\int_{-1}^{0} t d t+(R) \int_{0}^{1} \Delta t+\int_{1}^{3} t \Delta t,\right. \\
& =\left[\left.\frac{1}{2} t^{2}\right|_{-1} ^{0}+1+\left.2 t^{2}\right|_{1} ^{0}(t+1) d t+\int_{0}^{1} 2 \Delta t+\int_{1}^{3}\left(t^{2}+1\right) \Delta t\right] \\
& =\left[2 \frac{1}{2}, 6 \frac{1}{2}\right] .
\end{aligned}
$$

\section{Some inequalities for the interval Riemann delta integral}

We begin by recalling the notions of convexity on time scales.

Definition 4 (Dinu [17]) We say that $f:[a, b]_{\mathbb{T}} \rightarrow \mathbb{R}$ is a convex function if for all $x, y \in[a, b]_{\mathbb{T}}$ and $\alpha \in[0,1]$ we have

$f(\alpha x+(1-\alpha) y) \leq \alpha f(x)+(1-\alpha) f(y)$

for which $\alpha x+(1-\alpha) y \in[a, b]_{\mathbb{T}}$. If inequality (11) is reversed, then $f$ is said to be concave. If $f$ is both convex and concave, then $f$ is said to be affine. The set of all convex, concave and affine interval-valued functions are denoted by $S X\left([a, b]_{\mathbb{T}}, \mathbb{R}\right), S V\left([a, b]_{\mathbb{T}}, \mathbb{R}\right)$, and $S A\left([a, b]_{\mathbb{T}}, \mathbb{R}\right)$, respectively.

We can now introduce the concept of interval-valued convexity.

Definition 5 We say that $f:[a, b]_{\mathbb{T}} \rightarrow \mathbb{R}_{\mathcal{I}}$ is a convex interval-valued function if for all $x, y \in[a, b]_{\mathbb{T}}$ and $\alpha \in$ $(0,1)$ we have

$\alpha f(x)+(1-\alpha) f(y) \subseteq f(\alpha x+(1-\alpha) y)$ 
for which $\alpha x+(1-\alpha) y \in[a, b]_{\mathbb{T}}$. If the set inclusion (2) is reversed, then $f$ is said to be concave. If $f$ is both convex and concave, then $f$ is said to be affine. The set of all convex, concave and affine interval-valued functions are denoted by $S X\left([a, b]_{\mathbb{T}}, \mathbb{R}_{\mathcal{I}}\right), S V\left([a, b]_{\mathbb{T}}, \mathbb{R}_{\mathcal{I}}\right)$ and $S A\left([a, b]_{\mathbb{T}}, \mathbb{R}_{\mathcal{I}}\right)$, respectively.

Remark 3 It is clear that if $\mathbb{T}=\mathbb{R}$, then Definition 5 implies the definition of convexity introduced by Breckner 7 .

Theorem 5 Let $f:[a, b]_{\mathbb{T}} \rightarrow \mathbb{R}_{\mathcal{I}}$ be such that

$$
f(t)=[\underline{f}(t), \bar{f}(t)]
$$

for all $t \in[a, b]_{\mathbb{T}}$. Then,

(1) $f \in S X\left([a, b]_{\mathbb{T}}, \mathbb{R}_{\mathcal{I}}\right)$ if and only if $\underline{f} \in S X\left([a, b]_{\mathbb{T}}, \mathbb{R}\right)$ and $\bar{f} \in S V\left([a, b]_{\mathbb{T}}, \mathbb{R}\right)$,

(2) $f \in S V\left([a, b]_{\mathbb{T}}, \mathbb{R}_{\mathcal{I}}\right)$ if and only if $\underline{f} \in S V\left([a, b]_{\mathbb{T}}, \mathbb{R}\right)$ and $\bar{f} \in S X\left([a, b]_{\mathbb{T}}, \mathbb{R}\right)$,

(3) $f \in S A\left([a, b]_{\mathbb{T}}, \mathbb{R}_{\mathcal{I}}\right)$ if and only if $\underline{f}, \bar{f} \in S A\left([a, b]_{\mathbb{T}}, \mathbb{R}\right)$.

Proof We only prove that part (1) of Theorem 5 holds. Suppose that $f \in S X\left([a, b]_{\mathbb{T}}, \mathbb{R}_{\mathcal{I}}\right)$ and consider $x, y \in$ $[a, b]_{\mathbb{T}}, \alpha \in[0,1]$. Then,

$$
\alpha f(x)+(1-\alpha) f(y) \subseteq f(\alpha x+(1-\alpha) y),
$$

that is,

$$
\begin{aligned}
& {[\alpha \underline{f}(x)+(1-\alpha) \underline{f}(y), \alpha \bar{f}(x)+(1-\alpha) \bar{f}(y)]} \\
& \subseteq[\underline{f}(\alpha x+(1-\alpha) y), \bar{f}(\alpha x+(1-\alpha) y)] .
\end{aligned}
$$

It follows that

$$
\alpha \underline{f}(x)+(1-\alpha) \underline{f}(y) \geq \underline{f}(\alpha x+(1-\alpha) y)
$$

and

$$
\alpha \bar{f}(x)+(1-\alpha) \bar{f}(y) \leq \bar{f}(\alpha x+(1-\alpha) y) .
$$

This shows that

$$
\underline{f} \in S X\left([a, b]_{\mathbb{T}}, \mathbb{R}\right) \text { and } \bar{f} \in S V\left([a, b]_{\mathbb{T}}, \mathbb{R}\right) .
$$

Conversely, if

$$
\underline{f} \in S X\left([a, b]_{\mathbb{T}}, \mathbb{R}\right) \text { and } \bar{f} \in S V\left([a, b]_{\mathbb{T}}, \mathbb{R}\right),
$$

by Definition [5 and the set inclusion (3), we have $f \in S X\left([a, b]_{\mathbb{T}}, \mathbb{R}_{\mathcal{I}}\right)$.

Theorem 6 (Dinu [17]) A convex function on $[a, b]_{\mathbb{T}}$ is continuous on $(a, b)_{\mathbb{T}}$.

Theorem 7 Let $f:[a, b]_{\mathbb{T}} \rightarrow \mathbb{R}_{\mathcal{I}}$ be such that

$$
f(t)=[\underline{f}(t), \bar{f}(t)]
$$

for all $t \in[a, b]_{\mathbb{T}}$. If

$f \in S X\left([a, b]_{\mathbb{T}}, \mathbb{R}_{\mathcal{I}}\right) \cup S V\left([a, b]_{\mathbb{T}}, \mathbb{R}_{\mathcal{I}}\right) \cup S A\left([a, b]_{\mathbb{T}}, \mathbb{R}_{\mathcal{I}}\right)$, then $f \in \mathcal{I R}_{\left(\Delta,[a, b]_{\mathbb{T}}\right)}$.
Proof Suppose that

$f \in S V\left([a, b]_{\mathbb{T}}, \mathbb{R}_{\mathcal{I}}\right) \cup S V\left([a, b]_{\mathbb{T}}, \mathbb{R}_{\mathcal{I}}\right) \cup S A\left([a, b]_{\mathbb{T}}, \mathbb{R}_{\mathcal{I}}\right)$.

Due to Theorems [5] and [6] it follows that $f$ and $\bar{f}$ are continuous. Then, from Theorem 5.19 of $\overline{6}$, we have that

$$
\bar{f}(t), \underline{f}(t) \in \mathcal{R}_{\left(\Delta,[a, b]_{\mathbb{T}}\right)} .
$$

Hence, $f \in \mathcal{I R}_{\left(\Delta,[a, b]_{\mathbb{T}}\right)}$.

Theorem 8 (Wong et al. [36]) Let $a, b \in[a, b]_{\mathbb{T}}$ and $c, d \in \mathbb{R}$. Suppose that $g \in C_{r d}\left([a, b]_{\mathbb{T}},(c, d)\right)$ and $h \in$ $C_{r d}\left([a, b]_{\mathbb{T}}, \mathbb{R}\right)$ with

$$
\int_{a}^{b}|h(s)| \Delta s>0
$$

If $f \in C((c, d), \mathbb{R})$ is convex, then

$f\left(\frac{\int_{a}^{b}|h(s)| g(s) \Delta s}{\int_{a}^{b}|h(s)| \Delta s}\right) \leq \frac{\int_{a}^{b}|h(s)| f(g(s)) \Delta s}{\int_{a}^{b}|h(s)| \Delta s}$.

If $f$ is concave, then inequality (4) is reversed.

\section{Theorem 9 (Jensen's inequality)}

Let $g \in C_{r d}\left([a, b]_{\mathbb{T}},(c, d)\right)$ and $h \in C_{r d}\left([a, b]_{\mathbb{T}}, \mathbb{R}\right)$ with

$$
\int_{a}^{b}|h(s)| \Delta s>0
$$

If $f \in C\left((c, d), \mathbb{R}_{\mathcal{I}}^{+}\right)$is a convex function, then

$$
\frac{(I R) \int_{a}^{b}|h(s)| f(g(s)) \Delta s}{\int_{a}^{b}|h(s)| \Delta s} \subseteq f\left(\frac{\int_{a}^{b}|h(s)| g(s) \Delta s}{\int_{a}^{b}|h(s)| \Delta s}\right) .
$$

Proof By hypothesis, we have

$$
|h| \overline{f(g)}, \quad|h| \underline{f(g)} \in \mathcal{R}_{(\Delta,[a, b])} .
$$

Hence, $|h| f(g) \in \mathcal{I R}_{(\Delta,[a, b])}$ and

$$
\begin{aligned}
& (I R) \int_{a}^{b}|h(s)| f(g(s)) \Delta s \\
& =\left[\int_{a}^{b}|h(s)| \underline{f(g)}(s) \Delta s, \int_{a}^{b}|h(s)| \overline{f(g)}(s) \Delta s\right] .
\end{aligned}
$$

From Theorem 8, it follows that

$$
\underline{f}\left(\frac{\int_{a}^{b}|h(s)| g(s) \Delta s}{\left(\int_{a}^{b}|h(s)| \Delta s\right.}\right) \leq \frac{\int_{a}^{b}|h(s)| \underline{f(g)}(s) \Delta s}{\int_{a}^{b}|h(s)| \Delta s}
$$

and

$$
\bar{f}\left(\frac{\int_{a}^{b}|h(s)| g(s) \Delta s}{\int_{a}^{b}|h(s)| \Delta s}\right) \geq \frac{\int_{a}^{b}|h(s)| \overline{f(g)}(s) \Delta s}{\int_{a}^{b}|h(s)| \Delta s},
$$


which implies

$$
\begin{aligned}
& {\left[\frac{\int_{a}^{b}|h(s)| \underline{f(g)}(s) \Delta s}{\int_{a}^{b}|h(s)| \Delta s}, \frac{\int_{a}^{b}|h(s)| \overline{f(g)}(s) \Delta s}{\int_{a}^{b}|h(s)| \Delta s}\right]} \\
& \subseteq\left[\underline{f}\left(\frac{\int_{a}^{b}|h(s)| g(s) \Delta s}{\int_{a}^{b}|h(s)| \Delta s}\right), \bar{f}\left(\frac{\int_{a}^{b}|h(s)| g(s) \Delta s}{\int_{a}^{b}|h(s)| \Delta s}\right)\right],
\end{aligned}
$$

that is,

$$
\begin{aligned}
& \frac{\left[\int_{a}^{b}\left|h(s) \underline{\mid f(g)}(s) \Delta s, \int_{a}^{b}\right| h(s) \mid \overline{f(g)}(s) \Delta s\right]}{\int_{a}^{b}|h(s)| \Delta s} \\
& \subseteq\left[\frac{f}{\subseteq}\left(\frac{\int_{a}^{b}|h(s)| g(s) \Delta s}{\int_{a}^{b}|h(s)| \Delta s}\right), \bar{f}\left(\frac{\int_{a}^{b}|h(s)| g(s) \Delta s}{\int_{a}^{b}|h(s)| \Delta s}\right)\right] .
\end{aligned}
$$

Finally, we obtain

$$
\frac{(I R) \int_{a}^{b}|h(s)| f(g(s)) \Delta s}{\int_{a}^{b}|h(s)| \Delta s} \subseteq f\left(\frac{\int_{a}^{b}|h(s)| g(s) \Delta s}{\int_{a}^{b}|h(s)| \Delta s}\right)
$$

The proof is complete.

Example 5 Suppose that $[a, b]_{\mathbb{T}}=[0,1] \cup\left\{\frac{3}{2}\right\}$, where $[0,1]$ is a real-valued interval. Let $g(s)=s^{2}, h(s)=e^{s}$, and $f(s)=\left[s^{2}, 4 \sqrt{s}\right]$. Then

$$
\begin{aligned}
& \frac{(I R) \int_{a}^{b}|h(s)| f(g(s)) \Delta s}{\int_{a}^{b}|h(s)| \Delta s} \\
& =\frac{(I R) \int_{0}^{\frac{3}{2}}\left[s^{4} e^{s}, 4 s e^{s}\right] \Delta s}{\int_{0}^{\frac{3}{2}} e^{s} \Delta s} \\
& =\frac{\left[\int_{0}^{\frac{3}{2}} s^{4} e^{s} \Delta s, \int_{0}^{\frac{3}{2}} 4 s e^{s} \Delta s\right]}{\int_{0}^{\frac{3}{2}} e^{s} \Delta s} \\
& =\frac{\left[\int_{0}^{1} s^{4} e^{s} d s+\int_{1}^{\frac{3}{2}} s^{4} e^{s} \Delta s, \int_{0}^{1} 4 s e^{s} d s+\int_{1}^{\frac{3}{2}} 4 s e^{s} \Delta s\right]}{\int_{0}^{1} e^{s} d s+\int_{1}^{\frac{3}{2}} e^{s} \Delta s} \\
& =\frac{\left[9 \frac{1}{2} e-24,4+2 e\right]}{\frac{3}{2} e-1} \\
& =\left[\frac{19 e-48}{3 e-2}, \frac{8+4 e}{3 e-2}\right],
\end{aligned}
$$

and

$$
\begin{aligned}
& f\left(\frac{\int_{a}^{b}|h(s)| g(s) \Delta s}{\int_{a}^{b}|h(s)| \Delta s}\right) \\
& =f\left(\frac{\int_{0}^{\frac{3}{2}} s^{2} e^{s} \Delta s}{\int_{0}^{\frac{3}{2}} e^{s} \Delta s}\right) \\
& =f\left(\frac{\int_{0}^{1} s^{2} e^{s} d s+\int_{1}^{\frac{3}{2}} s^{2} e^{s} \Delta s}{\frac{3}{2} e-1}\right) \\
& \left.=f\left(\frac{\frac{3}{2} e-2}{\frac{3}{2} e-1}\right)^{s}\right) \\
& =\left[\left(\frac{3 e-4}{3 e-2}\right)^{2}, 4 \sqrt{\frac{3 e-4}{3 e-2}}\right] .
\end{aligned}
$$

It follows that

$$
\left[\frac{19 e-48}{3 e-2}, \frac{8+4 e}{3 e-2}\right] \subseteq\left[\left(\frac{3 e-4}{3 e-2}\right)^{2}, 4 \sqrt{\frac{3 e-4}{3 e-2}}\right] .
$$

It is clear that if $[a, b]_{\mathbb{T}}=[0,1]$ and $h(s) \equiv 1$, then we get a similar result given in [13, Theorem 3.5] by T. M. Costa. Similarly, we can get the following results that generalize [13, Theorem 3.4] and [13, Corollary 3.3].

Theorem 10 Let $g \in C_{r d}\left([a, b]_{\mathbb{T}},(c, d)\right)$ and $h \in C_{r d}\left([a, b]_{\mathbb{T}}, \mathbb{R}\right)$ with

$$
\int_{a}^{b}|h(s)| \Delta s>0 .
$$

If $f \in C\left((c, d), \mathbb{R}_{\mathcal{I}}^{+}\right)$is a concave function, then

$$
\frac{(I R) \int_{a}^{b}|h(s)| f(g(s)) \Delta s}{\int_{a}^{b}|h(s)| \Delta s} \supseteq f\left(\frac{\int_{a}^{b}|h(s)| \underline{f(g)}(s) \Delta s}{\int_{a}^{b}|h(s)| \Delta s}\right) .
$$

Theorem 11 Let $g \in C_{r d}\left([a, b]_{\mathbb{T}},(c, d)\right)$ and $h \in C_{r d}\left([a, b]_{\mathbb{T}}, \mathbb{R}\right)$ with

$$
\int_{a}^{b}|h(s)| \Delta s>0
$$

If $f \in C\left((c, d), \mathbb{R}_{\mathcal{I}}^{+}\right)$is an affine function, then

$$
\frac{(I R) \int_{a}^{b}|h(s)| f(g(s)) \Delta s}{\int_{a}^{b}|h(s)| \Delta s}=f\left(\frac{\int_{a}^{b}|h(s)| \underline{f(g)}(s) \Delta s}{\int_{a}^{b}|h(s)| \Delta s}\right) .
$$

Theorem 12 (Agarwal et al. [1])

Let $f, g, h \in C_{r d}\left([a, b]_{\mathbb{T}},(0, \infty)\right)$. If $\frac{1}{p}+\frac{1}{q}=1$, with $p>1$, then

$$
\begin{aligned}
& \int_{a}^{b} h(s) f(s) g(s) \Delta s \\
& \leq\left(\int_{a}^{b} h(s) f^{p}(s) \Delta s\right)^{\frac{1}{p}}\left(\int_{a}^{b} h(s) g^{q}(s) \Delta s\right)^{\frac{1}{q}} .
\end{aligned}
$$


Next we present a Hölder type inequality for intervalvalued functions on time scales.

\section{Theorem 13 (Hölder's inequality)}

Let $h \in C_{r d}\left([a, b]_{\mathbb{T}},(0, \infty)\right), f, g \in C_{r d}\left([a, b]_{\mathbb{T}}, \mathbb{R}_{\mathcal{I}}^{+}\right)$.

If $\frac{1}{p}+\frac{1}{q}=1$, with $p>1$, then

$$
\begin{aligned}
& \int_{a}^{b} h(s) f(s) g(s) \Delta s \\
& \leq\left(\int_{a}^{b} h(s) f^{p}(s) \Delta s\right)^{\frac{1}{p}}\left(\int_{a}^{b} h(s) g^{q}(s) \Delta s\right)^{\frac{1}{q}}
\end{aligned}
$$

Proof By hypothesis, we have

$$
\begin{aligned}
& \int_{a}^{b} h(s) f(s) g(s) \Delta s \\
& =\int_{a}^{b} h(s)[\underline{f}(s) \underline{g}(s), \bar{f}(s) \bar{g}(s)] \Delta s \\
& =\left[\int_{a}^{b} h(s) \underline{f}(s) \underline{g}(s) \Delta s, \int_{a}^{b} h(s) \bar{f}(s) \bar{g}(s) \Delta s\right] \\
& \leq\left[\left(\int_{a}^{b} h(s) \underline{f}^{p}(s) \Delta s\right)^{\frac{1}{p}}\left(\int_{a}^{b} h(s) \underline{g}^{q}(s) \Delta s\right)^{\frac{1}{q}},\right. \\
& \left.\left(\int_{a}^{b} h(s) \bar{f}^{p}(s) \Delta s\right)^{\frac{1}{p}}\left(\int_{a}^{b} h(s) \bar{g}^{q}(s) \Delta s\right)^{\frac{1}{q}}\right] \\
& =\left[\left(\int_{a}^{b} h(s) \underline{f}^{p}(s) \Delta s\right)^{\frac{1}{p}},\left(\int_{a}^{b} h(s) \bar{f}^{p}(s) \Delta s\right)^{\frac{1}{p}}\right] \\
& \text {. }\left[\left(\int_{a}^{b} h(s) \underline{g}^{q}(s) \Delta s\right)^{\frac{1}{q}},\left(\int_{a}^{b} h(s) \bar{g}^{q}(s) \Delta s\right)^{\frac{1}{q}}\right] \\
& =\left[\int_{a}^{b} h(s) \underline{f}^{p}(s) \Delta s, \int_{a}^{b} h(s) \bar{f}^{p}(s) \Delta s\right]^{\frac{1}{p}} \\
& {\left[\int_{a}^{b} h(s) \underline{g}^{q}(s) \Delta s, \int_{a}^{b} h(s) \bar{g}^{q}(s) \Delta s\right]^{\frac{1}{q}}} \\
& =\left(\int_{a}^{b} h(s)[\underline{f}(s), \bar{f}(s)]^{p} \Delta s\right)^{\frac{1}{p}}\left(\int_{a}^{b} h(s)[\underline{g}(s), \bar{g}(s)]^{q} \Delta s\right)^{\frac{1}{q}} \\
& =\left(\int_{a}^{b} h(s) f^{p}(s) \Delta s\right)^{\frac{1}{p}}\left(\int_{a}^{b} h(s) g^{q}(s) \Delta s\right)^{\frac{1}{q}} \text {. }
\end{aligned}
$$

This concludes the proof.

For the particular case $p=q=2$ in Theorem 13 . we obtain the following Cauchy-Schwarz inequality.
Theorem 14 (Cauchy-Schwarz inequality)

Let $h \in C_{r d}\left([a, b]_{\mathbb{T}},(0, \infty)\right), f, g \in C_{r d}\left([a, b]_{\mathbb{T}}, \mathbb{R}_{\mathcal{I}}^{+}\right)$. Then,

$$
\begin{aligned}
& \int_{a}^{b} h(s) f(s) g(s) \Delta s \\
& \leq \sqrt{\left(\int_{a}^{b} h(s) f^{2}(s) \Delta s\right)\left(\int_{a}^{b} h(s) g^{2}(s) \Delta s\right)} .
\end{aligned}
$$

Example 6 Suppose that $[a, b]_{\mathbb{T}}=\left[0, \frac{\pi}{2}\right]$. Let $h(s)=s$, $f(s)=[s, s+1]$, and $g(s)=[\sin s, s]$ for $s \in\left[0, \frac{\pi}{2}\right]$. Then

$$
\begin{array}{rl}
\int_{a}^{b} & h(s) f(s) g(s) \Delta s \\
& =\int_{0}^{\frac{\pi}{2}}\left[s^{2} \sin s, s^{3}+s^{2}\right] \Delta s \\
& =\left[\int_{0}^{\frac{\pi}{2}} s^{2} \sin s \Delta s, \int_{0}^{\frac{\pi}{2}}\left(s^{3}+s^{2}\right) \Delta s\right] \\
& =\left[\pi-2, \frac{\pi^{4}}{64}+\frac{\pi^{3}}{24}\right],
\end{array}
$$

and

$$
\begin{aligned}
& \sqrt{\left(\int_{a}^{b} h(s) f^{2}(s) \Delta s\right)\left(\int_{a}^{b} h(s) g^{2}(s) \Delta s\right)} \\
& =\sqrt{\left(\int_{0}^{\frac{\pi}{2}}\left[s^{3}, s^{3}+2 s^{2}+s\right] \Delta s\right)\left(\int_{0}^{\frac{\pi}{2}}\left[s \sin ^{2} s, s^{3}\right] \Delta s\right)} \\
& =\sqrt{\left[\int_{0}^{\frac{\pi}{2}} s^{3} d s, \int_{0}^{\frac{\pi}{2}}\left(s^{3}+2 s^{2}+s\right) d s\right] \cdot\left[\int_{0}^{\frac{\pi}{2}} s \sin ^{2} s d s, \int_{0}^{\frac{\pi}{2}} s^{3} d s\right]} \\
& =\sqrt{\left[\frac{\pi^{4}}{64}, \frac{\pi^{4}}{64}+\frac{\pi^{3}}{12}+\frac{\pi^{2}}{8}\right] \cdot\left[\frac{\pi^{2}}{16}+\frac{1}{4}, \frac{\pi^{4}}{64}\right]} \\
& =\sqrt{\left[\frac{\pi^{6}}{1024}+\frac{\pi^{4}}{256}, \frac{\pi^{8}}{4096}+\frac{\pi^{7}}{768}+\frac{\pi^{6}}{512}\right]} \\
& =\left[\sqrt{\frac{\pi^{6}}{1024}+\frac{\pi^{4}}{256}}, \sqrt{\frac{\pi^{8}}{4096}+\frac{\pi^{7}}{768}+\frac{\pi^{6}}{512}}\right]
\end{aligned}
$$$$
\text { Consequently, we obtain }
$$$$
\begin{aligned}
& {\left[\pi-2, \frac{\pi^{4}}{64}+\frac{\pi^{3}}{24}\right]} \\
& \leq\left[\sqrt{\frac{\pi^{6}}{1024}+\frac{\pi^{4}}{256}}, \sqrt{\frac{\pi^{8}}{4096}+\frac{\pi^{7}}{768}+\frac{\pi^{6}}{512}}\right] .
\end{aligned}
$$

Example 7 Suppose that $[a, b]_{\mathbb{T}}=\{0,1,2,3\}$. Let $h(s)=$ $s, f(s)=[s, s+1]$, and $g(s)=\left[\frac{s}{2}, s\right]$ for $s \in\{0,1,2,3\}$. 
Some inequalities for interval-valued functions on time scales

9

Then

$$
\begin{array}{rl}
\int_{a}^{b} & h(s) f(s) g(s) \Delta s \\
& =\int_{0}^{3}\left[\frac{s^{3}}{2}, s^{3}+s^{2}\right] \Delta s \\
& =\left[\int_{0}^{3} \frac{s^{3}}{2} \Delta s, \int_{0}^{3} s^{3}+s^{2} \Delta s\right] \\
& =\left[\frac{9}{2}, 14\right]
\end{array}
$$

and

$$
\begin{aligned}
& \sqrt{\left(\int_{a}^{b} h(s) f^{2}(s) \Delta s\right)\left(\int_{a}^{b} h(s) g^{2}(s) \Delta s\right)} \\
& =\sqrt{\left(\int_{0}^{3}\left[s^{3}, s^{3}+2 s^{2}+s\right] \Delta s\right)\left(\int_{0}^{3}\left[\frac{s^{3}}{4}, s^{3}\right] \Delta s\right)} \\
& =\sqrt{[9,22] \cdot\left[\frac{9}{4}, 9\right]} \\
& =\left[\frac{9}{2}, 3 \sqrt{22}\right] .
\end{aligned}
$$

Consequently, we obtain

$$
\left[\frac{9}{2}, 14\right] \leq\left[\frac{9}{2}, 3 \sqrt{22}\right]
$$

Theorem 15 (Agarwal et al.[1]; Wong et al.[37])

Let $f, g, h \in C_{r d}\left([a, b]_{\mathbb{T}}, \mathbb{R}\right)$ and $p>1$. Then,

$$
\begin{aligned}
& \left(\int_{a}^{b}|h(s)||f(s)+g(s)|^{p} \Delta s\right)^{\frac{1}{p}} \\
& \leq\left(\int_{a}^{b}|h(s)||f(s)|^{p} \Delta s\right)^{\frac{1}{p}}+\left(\int_{a}^{b}|h(s)||g(s)|^{p} \Delta s\right)^{\frac{1}{p}} .
\end{aligned}
$$

By the same technique used in the proof of Thearem 4 in 32, we get a more general result.

Theorem 16 (Minkowski's inequality)

Let $h \in C_{r d}\left([a, b]_{\mathbb{T}}, \mathbb{R}\right), f, g \in C\left([a, b]_{\mathbb{T}}, \mathbb{R}_{\mathcal{I}}^{+}\right)$and $p>1$. Then,

$$
\begin{array}{ll}
\left(\int_{a}^{b}|h(s)|(f(s)+g(s))^{p} \Delta s\right)^{\frac{1}{p}} & =\sqrt{\left[\int_{0}^{2} 4 s^{3} \Delta s, \int_{0}^{2} s e^{2 s}+4 s^{2} e^{s}+4 s^{3} \Delta s\right]} \\
\leq\left(\int_{a}^{b}|h(s)| f^{p}(s) \Delta s\right)^{\frac{1}{p}}+\left(\int_{a}^{b}|h(s)| g^{p}(s) \Delta s\right)^{\frac{1}{p}} . & =\left[\sqrt{5}, \frac{\sqrt{5 e^{2}+32 e-11}}{2}\right],
\end{array}
$$

Proof By hypothesis, we have

$$
\begin{aligned}
& \left(\int_{a}^{b}|h(s)|(f(s)+g(s))^{p} \Delta s\right)^{\frac{1}{p}} \\
& \left.=\left(\int_{a}^{b}|h(s)| \underline{f}(s)+\underline{g}(s), \bar{f}(s)+\bar{g}(s)\right]^{p} \Delta s\right)^{\frac{1}{p}} \\
& =\left(\int_{a}^{b}|h(s)|\left[(\underline{f}(s)+\underline{g}(s))^{p},(\bar{f}(s)+\bar{g}(s))^{p}\right] \Delta s\right)^{\frac{1}{p}} \\
& =\left[\left(\int_{a}^{b}|h(s)|(\underline{f}(s)+\underline{g}(s))^{p} \Delta s\right)^{\frac{1}{p}},\right. \\
& \left.\left(\int_{a}^{b}|h(s)|(\bar{f}(s)+\bar{g}(s))^{p} \Delta s\right)^{\frac{1}{p}}\right] \\
& \leq\left[\left(\int_{a}^{b}|h(s)| \underline{f}^{p}(s) \Delta s\right)^{\frac{1}{p}}+\left(\int_{a}^{b}|h(s)| \underline{g}^{p}(s) \Delta s\right)^{\frac{1}{p}},\right. \\
& \left.\left(\int_{a}^{b}|h(s)| \bar{f}^{p}(s) \Delta s\right)^{\frac{1}{p}}+\left(\int_{a}^{b}|h(s)| \bar{g}^{p}(s) \Delta s\right)^{\frac{1}{p}}\right] \\
& =\left[\left(\int_{a}^{b}|h(s)| \underline{f}^{p}(s) \Delta s\right)^{\frac{1}{p}},\left(\int_{a}^{b}|h(s)| \bar{f}^{p}(s) \Delta s\right)^{\frac{1}{p}}\right] \\
& +\left[\left(\int_{a}^{b}|h(s)| \underline{g}^{p}(s) \Delta s\right)^{\frac{1}{p}},\left(\int_{a}^{b}|h(s)| \bar{g}^{p}(s) \Delta s\right)^{\frac{1}{p}}\right] \\
& =\left[\int_{a}^{b}|h(s)| \underline{f}^{p}(s) \Delta s, \int_{a}^{b}|h(s)| \bar{f}^{p}(s) \Delta s\right]^{\frac{1}{p}} \\
& +\left[\int_{a}^{b}|h(s)| \underline{g}^{p}(s) \Delta s, \int_{a}^{b}|h(s)| \bar{g}^{p}(s) \Delta s\right]^{\frac{1}{p}} \\
& =\left(\int_{a}^{b}|h(s)|[\underline{f}(s), \bar{f}(s)]^{p} \Delta s\right)^{\frac{1}{p}} \\
& +\left(\int_{a}^{b}|h(s)|[\underline{g}(s), \bar{g}(s)]^{p} \Delta s\right)^{\frac{1}{p}} \\
& =\left(\int_{a}^{b}|h(s)| f^{p}(s) \Delta s\right)^{\frac{1}{p}}+\left(\int_{a}^{b}|h(s)| g^{p}(s) \Delta s\right)^{\frac{1}{p}} .
\end{aligned}
$$

The proof is complete.

Example 8 Suppose that $[a, b]_{\mathbb{T}}=[0,1] \cup\{2\}$. Let $h(s)=s, f(s)=[s, 2 s], g(s)=\left[s, e^{s}\right]$ and $p=2$. Then, $\left(\int_{a}^{b}|h(s)|(f(s)+g(s))^{p} \Delta s\right)^{\frac{1}{p}}$

$=\sqrt{\int_{0}^{2}\left[4 s^{3}, s e^{2 s}+4 s^{2} e^{s}+4 s^{3}\right] \Delta s}$ 
and

$$
\begin{aligned}
& \left(\int_{a}^{b}|h(s)| f^{p}(s) \Delta s\right)^{\frac{1}{p}}+\left(\int_{a}^{b}|h(s)| g\left({ }^{p} s\right) \Delta s\right)^{\frac{1}{p}} \\
& =\sqrt{\int_{0}^{2}\left[s^{3}, 4 s^{3}\right] \Delta s}+\sqrt{\int_{0}^{2}\left[s^{3}, s e^{2 s}\right] \Delta s} \\
& =\left[\frac{\sqrt{5}}{2}, \sqrt{5}\right]+\left[\frac{\sqrt{5}}{2}, \frac{\sqrt{5 e^{2}+1}}{2}\right] \\
& =\left[\sqrt{5}, \sqrt{5}+\frac{\sqrt{5 e^{2}+1}}{2}\right] .
\end{aligned}
$$

Consequently, we obtain

$$
\left[\sqrt{5}, \frac{\sqrt{5 e^{2}+32 e-11}}{2}\right] \leq\left[\sqrt{5}, \sqrt{5}+\frac{\sqrt{5 e^{2}+1}}{2}\right] .
$$

The next results follow directly from Theorems 13 and [16, respectively.

Corollary 1 Let $h \in C_{r d}\left([a, b]_{\mathbb{T}},(0, \infty)\right)$, and $f, g \in C_{r d}\left([a, b]_{\mathbb{T}}, \mathbb{R}_{\mathcal{I}}^{-}\right)$. If $\frac{1}{p}+\frac{1}{q}=1$, with $p>1$, then

$$
\begin{aligned}
& \int_{a}^{b} h(s) f(s) g(s) \Delta s \\
& \leq\left(\int_{a}^{b} h(s)(-f)^{p}(s) \Delta s\right)^{\frac{1}{p}}\left(\int_{a}^{b} h(s)(-g)^{q}(s) \Delta s\right)^{\frac{1}{q}} .
\end{aligned}
$$

Corollary 2 Let $h \in C_{r d}\left([a, b]_{\mathbb{T}}, \mathbb{R}\right), f, g \in C\left([a, b]_{\mathbb{T}}, \mathbb{R}_{\mathcal{I}}^{-}\right)$ and $p \in 2^{\mathbb{N}}$. Then,

$$
\begin{aligned}
& \left(\int_{a}^{b}|h(s)|(f(s)+g(s))^{p} \Delta s\right)^{\frac{1}{p}} \\
& \leq\left(\int_{a}^{b}|h(s)| f^{p}(s) \Delta s\right)^{\frac{1}{p}}+\left(\int_{a}^{b}|h(s)| g^{p}(s) \Delta s\right)^{\frac{1}{p}} .
\end{aligned}
$$

\section{Conclusion}

We investigated Darboux and Riemann interval delta integrals for interval-valued functions on time scales. Inequalities for interval-valued functions were proved. Our results generalize previous inequalities presented by Costa [13, Corollary 3.3, Theorem 3.4, Theorem 3.5] and Román-Flores [32, Theorem 4].

Acknowledgements This study was funded by Fundamental Research Funds for the Central Universities (Grant Numbers 2017B19714 and 2017B07414). Torres was supported by FCT and CIDMA, project UID/MAT/04106/2013.

The authors are very grateful to two anonymous referees, for several valuable and helpful comments, suggestions and questions, which helped them to improve the paper into present form.

\section{Compliance with ethical standards}

Conflicts of interest The authors declare that they have no conflict of interest.

Ethical approval This article does not contain any studies with human participants or animals performed by any of the authors.

\section{References}

1. R. Agarwal, D. O'Regan, S. Saker, Dynamic inequalities on time scales, Springer, Cham, (2014).

2. B. Bede, Mathematics of Fuzzy Sets and Fuzzy Logic, Studies in Fuzziness and Soft Computing, 295, Springer, Heidelberg, (2013).

3. N. Benkhettou, A. M. C. Brito da Cruz, D. F. M. Torres, A fractional calculus on arbitrary time scales: fractional differentiation and fractional integration, Signal Process., 107 (2015), 230-237. arXiv:1405.2813

4. N. Benkhettou, A. M. C. Brito da Cruz, D. F. M. Torres, Nonsymmetric and symmetric fractional calculus on arbitrary nonempty closed sets, Math. Methods Appl. Sci. 39 (2016), 261-279. arXiv: 1502.07277

5. M. Bohner, A. Peterson, Dynamic equations on time scales: an introduction with applications, Birkhäuser, Boston, MA (2001).

6. M. Bohner, A. Peterson, Advances in dynamic equations on time scales, Birkhäuser, Boston, MA (2003).

7. W. W. Breckner, Continuity of generalized convex and generalized concave set-valued functions, Rev. Anal. Numér. Théor. Approx., 22 (1993), 39-51.

8. O. Caprani, K. Madsen, L. B. Rall, Integration of interval functions, SIAM J. Math. Anal., 12 (1981), 321-341.

9. Y. Chalco-Cano, A. Flores-Franulič, H. Román-Flores, Ostrowski type inequalities for interval-valued functions using generalized Hukuhara derivative, Comput. Appl. Math., 31 (2012), 457-472.

10. Y. Chalco-Cano, W. A. Lodwick, W. Condori-Equice, Ostrowski type inequalities and applications in numerical integration for interval-valued functions, Soft Comput., 19 (2015), 3293-3300.

11. Y. Chalco-Cano, A. Rufián-Lizana, H. Román-Flores, M. D. Jiménez-Gamero, Calculus for interval-valued functions using generalized Hukuhara derivative and applications, Fuzzy Sets and Systems, 219 (2013), 49-67.

12. Y. Chalco-Cano, G. N. Silva, A. Rufián-Lizana, On the Newton method for solving fuzzy optimization problems, Fuzzy Sets and Systems, 272 (2015), 60-69.

13. T. M. Costa, Jensen's inequality type integral for fuzzyinterval-valued functions, Fuzzy Sets and Systems, 327 (2017), 31-47.

14. T. M. Costa, H. Bouwmeester, W. A. Lodwick, C. Lavor, Calculating the possible conformations arising from uncertainty in the molecular distance geometry problem using constraint interval analysis, Inform. Sci., 415-416 (2017), $41-52$.

15. T. M. Costa, Y. Chalco-Cano, W. A. Lodwick, G. N. Silva, Generalized interval vector spaces and interval optimization, Inform. Sci., 311 (2015), 74-85.

16. T. M. Costa, H. Román-Flores, Some integral inequalities for fuzzy-interval-valued functions, Inform. Sci., 420 (2017), 110-125.

17. C. Dinu, Convex functions on time scales, An. Univ. Craiova Ser. Mat. Inform., 35 (2008), 87-96. 
18. O. S. Fard, T. A. Bidgoli, Calculus of fuzzy functions on time scales (I), Soft Comput., 19 (2015), 293-305.

19. O. S. Fard, D. F. M. Torres, M. R. Zadeh, A Hukuhara approach to the study of hybrid fuzzy systems on time scales, Appl. Anal. Discrete Math. 10 (2016), 152-167. arXiv: 1603.03737

20. A. Flores-Franulič, Y. Chalco-Cano, H. Román-Flores, An Ostrowski type inequality for interval-valued functions, IFSA World Congress and NAFIPS Annual Meeting IEEE, 35 (2013), 1459-1462.

21. N. A. Gasilov, S.. E. Amrahov, Solving a nonhomogeneous linear system of interval differential equations, Soft Comput., 22 (2018), 3817-3828.

22. S. Hilger, Ein Maßkettenkalkül mit Anwendung auf Zentrumsmannigfaltigkeiten, Ph.D. Thesis, Universität Würzburg (1988).

23. L. Jaulin, M. Kieffer, O. Didrit, É. Walter, Applied interval analysis, Springer-Verlag London, Ltd., London, (2001).

24. V. Lupulescu, Hukuhara differentiability of interval-valued functions and interval differential equations on time scales, Inform. Sci., 248 (2013), 50-67.

25. V. Lupulescu, Fractional calculus for interval-valued functions, Fuzzy Sets and Systems, 265 (2015), 63-85.

26. V. Lupulescu, N. V. Hoa, Interval Abel integral equation, Soft Comput., 21 (2017), 2777-2784.

27. R. E. Moore, Interval analysis, Prentice-Hall, Inc., Englewood Cliffs, N.J., (1966).

28. R. E. Moore, Methods and applications of interval analysis, SIAM, Philadelphia, Pa., (1979).

29. R. E. Moore, R. B. Kearfott, M. J. Cloud, Introduction to interval analysis, SIAM, Philadelphia, PA., (2009).

30. R. Osuna-Gómez, Y. Chalco-Cano, B. HernándezJiménez, G. Ruiz-Garzón, Optimality conditions for generalized differentiable interval-valued functions, Inform. Sci., 321 (2015), 136-146.

31. L. B. Rall, Integration of interval functions. II. The finite case, SIAM J. Math. Anal., 13 (1982), 690-697.

32. H. Román-Flores, Y. Chalco-Cano, W. A. Lodwick, Some integral inequalities for interval-valued functions, Comput. Appl. Math., 37 (2018), 1306-1318.

33. H. Román-Flores, Y. Chalco-Cano, G. N. Silva, A note on Gronwall type inequality for interval-valued functions, IFSA World Congress and NAFIPS Annual Meeting IEEE, 35 (2013), 1455-1458.

34. L. Stefanini, B. Bede, Generalized Hukuhara differentiability of interval-valued functions and interval differential equations, Nonlinear Anal., 71 (2009), 1311-1328.

35. C. Vasavi, G. S. Kumar, M. S. N. Murty, Generalized differentiability and integrability for fuzzy set-valued functions on time scales, Soft Comput., 20 (2016), 1093-1104.

36. F.-H. Wong, C.-C. Yeh, W.-C. Lian, An extension of Jensen's inequality on time scales, Adv. Dyn. Syst. Appl., 1 (2006), 113-120.

37. F.-H. Wong, C.-C. Yeh, S.-L. Yu, C.-H. Hong, Young's inequality and related results on time scales, Appl. Math. Lett., 18 (2005), 983-988.

38. C. X. Wu, Z. T. Gong, On Henstock integrals of intervalvalued functions and fuzzy-valued functions, Fuzzy Sets and Systems, 115 (2000), 377-391.

39. X. X. You, D. F. Zhao, On convergence theorem for the McShane integral on time scales, J. Chungcheong Math. Soc., 25 (2012), 393-400.

40. X. X. You, D. F. Zhao, D. F. M. Torres, On the HenstockKurzweil integral for Riesz-space-valued functions on time scales, J. Nonlinear Sci. Appl., 10 (2017), 2487-2500. arXiv: 1704.06808
41. D. F. Zhao, T. X. Li, On conformable delta fractional calculus on time scales, J. Math. Computer Sci., 16 (2016), 324-335.

42. P. Z. Zhou, J. B. Du, Z. H. LÜ, Interval analysis based robust truss optimization with continuous and discrete variables using mix-coded genetic algorithm, Struct. Multidiscip. Optim., 56 (2017), 353-370. 